\title{
Wer darf wann Cannabis verordnen?
}

_ Ärzte jeder Fachrichtung dürfen seit dem 1. März 2017 Cannabisblüten und -extrakte mittels Betäubungsmittel-Rezept zulasten der GKV verordnen. Diese Berechtigung ist mit keiner besonderen Qualifikation verbunden. Auch das komplizierte bisherige Genehmigungsverfahren ist entfallen. Die Patienten sind nicht mehr verpflichtet, eine Ausnahmeerlaubnis nach $\$ 3$ Abs. 2 Betäubungsmittelgesetz (BtMG) bei der Bundesopiumstelle des Bundesinstituts für Arzneimittel und Medizinprodukte (BfArM) zu beantragen, um eine standardisierte Cannabisextraktzubereitung oder MedizinalCannabisblüten zur Anwendung im Rahmen einer ärztlich begleiteten Selbsttherapie zu erwerben.

\section{MMW-KOMMENTAR}

Es handelt sich so gesehen um eine Verlagerung einer Tätigkeit aus einer Bundesbehörde in die Praxen der niedergelassenen Ärztenatürlich ohne ensprechende Vergütung. Die neue Aufgabe ist dabei keineswegs leicht zu bewältigen. Cannabisblüten und -extrakte können nämlich für jede Indikation verord-

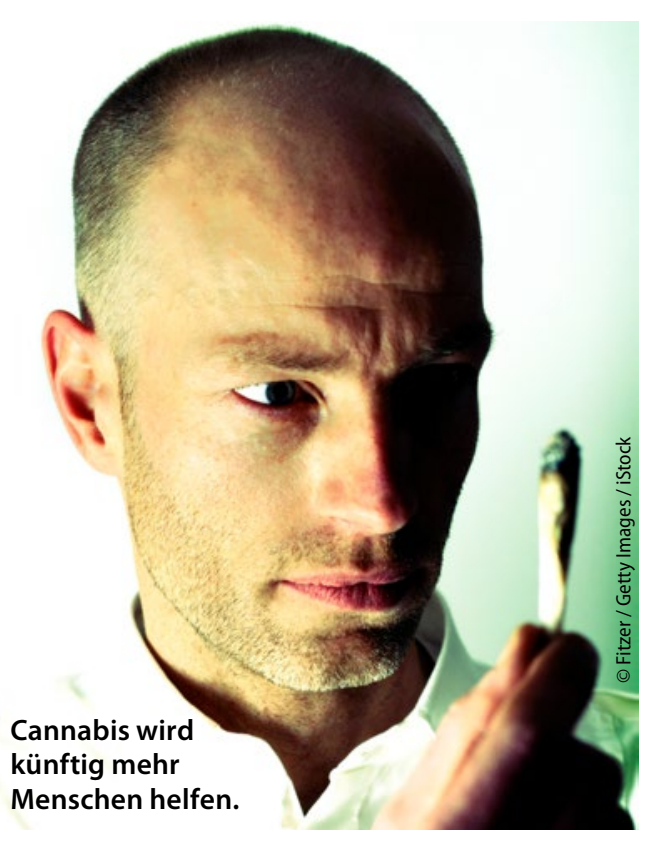

net werden, da im Gesetz ausdrücklich darauf verzichtet wurde, einzelne Indikationen aufzuführen. Die Indikationsstellung ist allgemein gehalten und kommt laut Gesetz immer dann zum Tragen, wenn

- "eine allgemein anerkannte, dem medizinischen Standard entsprechende Leistung

\section{Etablierte Indikationen für die Therapie mit Medizinal-Cannabis}

- chronische, insbesondere neuropathische Schmerzen

- Spastik bei multipler Sklerose (MS)

- Appetitlosigkeit

- Übelkeit und Erbrechen

\section{Hinweise für positive Wirkungen}

- neurologische Erkrankungen wie Spastik, Schmerzen oder hyperkinetische Bewegungsstörungen

- dermatologische Erkrankungen wie Neurodermitis, Psoriasis, Akne inversa oder Hyperhidrosis

- Glaukom

- internistische Erkrankungen wir Arthritis, Colitis ulcerosa oder Morbus Crohn

- psychiatrische Erkrankungen wie Depression, Angststörungen, posttraumatische Belastungsstörung, ADHS oder Schlafstörungen

im Einzelfall nicht zur Verfügung steht", oder alternativ

- diese Leistung „im Einzelfall nach der begründeten Einschätzung des behandelnden Vertragsarztes unter Abwägung der zu erwartenden Nebenwirkungen und unter Berücksichtigung des Krankheitszustandes der oder des Versicherten nicht zur Anwendung kommen kann".

Eine arzneimittelrechtliche Zulassung für Cannabisprodukte gibt es bisher nicht, was die Frage nach der Verordnungsgrundlage verkompliziert. Zurückgreifen kann man hier auf die mehr als 50 Erkrankungen bzw. Symptome, für die das BfArM in den Jahren 2007-2016 eine Ausnahmeerlaubnis für die Cannabistherapie erteilt hat (siehe Kasten). Beachtenswert ist aber, dass vor Behandlungsbeginn die Krankenkasse die Kostenübernahme genehmigen muss. Im Gesetz ist dabei festgelegt, dass die Kasse einen solchen Antrag ", nur in begründeten Ausnahmefällen" ablehnen darf. Auch soll der Antrag innerhalb von drei bis fünf Wochen entschieden sein.

Die Verschreibungshöchstmenge für Cannabis beträgt $100 \mathrm{~g}$ in 30 Tagen. Zwecks einfacherer Handhabbarkeit wurde die Höchstmenge unabhängig vom Gehalt einzelner Cannabinoide in der jeweiligen Cannabissorte festgelegt. Aktuell können auf dieser Grundlage 13 verschiedene Cannabissorten verordnet werden, die allesamt aus dem Ausland (Niederlande, Kanada) importiert werden. Sie alle sind auf den Gehalt der Cannabinoide THC und CBD standardisiert.

Ärzte, denen das alles momentan noch zu viel Aufwand ist, finden ein Hintertürchen im Gesetz. Wer Cannabis verordnet, muss nämlich an einer nicht-interventionellen, ausschließlich wissenschaftlichen Zwecken dienenden Begleiterhebung teilnehmen. Ist man dazu nicht bereit (oder z. B. zeitlich nicht in der Lage), ist die Kostenübernahme durch die Kasse ausgeschlossen. 